IJMMS 30:1 (2002) 1-8

PII. S0161171202012607

http://ijmms.hindawi.com

(c) Hindawi Publishing Corp.

\title{
A MEASURE OF GRAPH VULNERABILITY: SCATTERING NUMBER
}

\section{ALPAY KIRLANGIÇ}

Received 2 April 2001 and in revised form 20 August 2001

\begin{abstract}
The scattering number of a graph $G$, denoted $\operatorname{sc}(G)$, is defined by $\operatorname{sc}(G)=\max \{c(G-S)-$ $|S|: S \subseteq V(G)$ and $c(G-S) \neq 1\}$ where $c(G-S)$ denotes the number of components in $G-$ $S$. It is one measure of graph vulnerability. In this paper, general results on the scattering number of a graph are considered. Firstly, some bounds on the scattering number are given. Further, scattering number of a binomial tree is calculated. Also several results are given about binomial trees and graph operations.
\end{abstract}

2000 Mathematics Subject Classification: 05C40, 05 C85.

1. Introduction. In a communication network, vulnerability measures the resistance of the network to disruption of operation after the failure of certain stations or communication links. To measure vulnerability we have some parameters that are toughness, binding number, vertex integrity, and scattering number [5]. In this paper, we discuss the scattering number of a graph.

The scattering number of a graph $G$, denoted $\operatorname{sc}(G)$, was introduced in [4]. Formally the scattering number is defined by

$$
\operatorname{sc}(G)=\max \{c(G-S)-|S|: S \subseteq V(G), c(G-S) \neq 1\},
$$

where $c(G-S)$ denotes the number of components in $G-S$. A cutset $S$ of a graph $G$ fulfilling $\operatorname{sc}(G)=c(G-S)-|S|$ is said to be a scattering set. The problem "given a graph $G$, decide whether the scattering number is larger than zero" is NP-complete.

The scattering number of a graph is closely related to the toughness of a graph and to the existence of Hamilton cycles and paths. The toughness of a graph $G$, denoted $t(G)$, was defined by Chvátal [1]: for the complete graph $K_{n}$ we have $t\left(K_{n}\right)=\infty$; if $G$ is not complete, then $t(G)=\min \{|S| / c(G-S): S \subseteq V(G), c(G-S)>1\}$. A graph $G$ is said to be $t$-though if $t(G) \geq t$, that is, $|S| \geq t c(G-S)$ for any cutset $S$. It follows from the definitions that $t(G) \geq 1$ if and only if $\operatorname{sc}(G) \leq 0$ for any graph $G$ [3]. Moreover, Jung [4] calls the scattering number the "additive dual" of the toughness.

A Hamilton cycle in a graph $G$ is a cycle containing every vertex of $G$. Similarly, a Hamilton path in a graph $G$ is a path that contains every vertex of $G$. If a graph $G$ has a Hamilton cycle, then $\operatorname{sc}(G) \leq 0$; and if a graph $G$ has a Hamilton path, then $\operatorname{sc}(G) \leq 1$ [3].

The following theorem is given by Deogun et al. [3].

THEOREM 1.1. (a) The scattering number of a graph $G \operatorname{sc}(G) \geq \operatorname{sc}\left(G-V^{\prime}\right)-\left|V^{\prime}\right|$ holds for every subset $V^{\prime} \subseteq V(G)$ in any graph $G$; 
(b) $\operatorname{sc}(G) \geq \operatorname{sc}(G-v)-1$ for every vertex $v \in V(G)$ in any graph $G$;

(c) let $G$ be a connected graph. Then there is a vertex $v \in V(G)$ such that $\operatorname{sc}(G) \leq$ $\operatorname{sc}(G-v)-1$;

(d) for every connected graph $G \mathrm{sc}(G)=\max _{v \in V(G)} \operatorname{sc}(G-v)-1$.

The path cover number of a graph $G$ is the smallest number of disjoint paths covering the vertex set of $G$ and is denoted by $\pi(G)$. For the next theorem a short proof is given in [3] and this theorem was also proven by Lehel without using order-theoretic tools [6].

THEOREM 1.2 (see [3]). If $G$ is cocomparability graph, then $\pi(G)=\max (1, \operatorname{sc}(G))$.

Now we give some definitions.

DefinITION 1.3. The connectivity $\kappa=\kappa(G)$ of a graph $G$ is the minimum number of vertices whose removal results in a disconnected or trivial graph.

Definition 1.4. A subset $X$ of $V$ is called a covering of $G$ if every edge of $G$ has at least one end in $X$. A covering $X$ is a minimum covering if $G$ has no covering $X^{\prime}$ with $\left|X^{\prime}\right|<|X|$. The covering number, $\alpha(G)$, is the number of vertices in a minimum covering of $G$.

Definition 1.5. A subset $X$ of $V$ is called an independent set of $G$ if no two vertices of $X$ are adjacent in $G$. An independent set $X$ is maximum if $G$ has no independent set $X^{\prime}$ with $\left|X^{\prime}\right|>|X|$. The independence number of $G, \beta(G)$, is the number of vertices in a maximum independent set of $G$.

In Section 2, some bounds on the scattering number are given. Section 3 gives several results about the scattering number and graph operations.

2. Bounds. Firstly, we give two theorems showing the relation between the toughness and the scattering number.

THEOREM 2.1. If $t(G) \leq 0$, then $\operatorname{sc}(G) \leq(\alpha(G) / t(G))(1-t(G))$.

Proof. For any cutset $S$, we have

$$
t(G)=\min \left\{\frac{|S|}{c(G-S)}\right\}=\min \left\{1-\frac{c(G-S)-|S|}{c(G-S)}\right\}=1-\max \left\{\frac{c(G-S)-|S|}{c(G-S)}\right\},
$$

and so

$$
\max \left\{\frac{c(G-S)-|S|}{c(G-S)}\right\}=1-t(G) .
$$

Hence,

$$
\frac{c(G-S)-|S|}{c(G-S)} \leq 1-t(G) \Longrightarrow c(G-S)-|S| \leq(1-t(G)) c(G-S)
$$


On the other hand,

$$
t(G)=\min \left\{\frac{|S|}{c(G-S)}\right\} \Rightarrow \frac{|S|}{c(G-S)} \geq t(G), \quad c(G-S) \leq \frac{|S|}{t(G)}
$$

for every cutset $S$. Since $S$ is a cutset, we have $|S| \leq \alpha(G)$ and $c(G-S) \leq \alpha(G) / t(G)$. If $t(G) \leq 0$, then

$$
(1-t(G)) c(G-S) \leq \frac{\alpha(G)}{t(G)}(1-t(G))
$$

By (2.3) and (2.5),

$$
c(G-S)-|S| \leq \frac{\alpha(G)}{t(G)}(1-t(G)), \quad t(G) \leq 0,
$$

and so

$$
\operatorname{sc}(G)=\max _{S}\{c(G-S)-|S|\} \leq \frac{\alpha(G)}{t(G)}(1-t(G)) .
$$

The proof is completed.

THEOREM 2.2. If $t(G)>0$, then $\operatorname{sc}(G) \leq \alpha(G) / t(G)-\kappa(G)$.

Proof. Consider any cutset $S$. Then $\kappa(G) \leq|S| \leq \alpha(G)$, obviously. Since $t(G)=$ $\min \{|S| / c(G-S)\}$, we have $c(G-S) \leq|S| / t(G)$ and so

$$
c(G-S)-|S| \leq \frac{|S|}{t(G)}-|S| \leq \frac{\alpha(G)}{t(G)}-\kappa(G) .
$$

Hence

$$
\operatorname{sc}(G)=\max _{S}\{c(G-S)-|S|\} \leq \frac{\alpha(G)}{t(G)}-\kappa(G) .
$$

The proof is completed.

Next, we give two theorems containing the relation between some graph parameters and the scattering number.

THEOREM 2.3. If a graph $G$ does not contain graph $2 K_{2}$ as an induced subgraph, then

$$
\operatorname{sc}(G)= \begin{cases}\beta(G)-\alpha(G), & \text { if } \kappa(G)=\alpha(G), \\ \beta(G)-\alpha(G)+1, & \text { if } \alpha(G)=\kappa(G)+1 .\end{cases}
$$

Proof. If a graph $G$ does not contain graph $2 K_{2}$ as an induced subgraph, then we have $\alpha(G)=\kappa(G)$ or $\alpha(G)=\kappa(G)+1$. That is, $|S|$ must be $\alpha(G)$ or $\alpha(G)-1$.

$$
\begin{aligned}
& \text { If }|S|=\alpha(G), \quad \text { then } c(G-S)=\beta(G), \\
& \text { If }|S|=\alpha(G)-1, \quad \text { then } c(G-S)=\beta(G) .
\end{aligned}
$$

By (2.11) the proof is completed. 


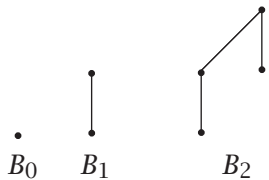

$B_{2}$

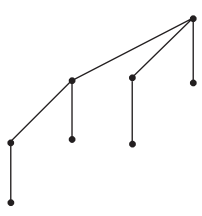

$B_{3}$

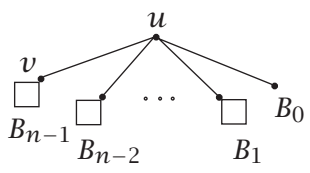

$B_{n}$

FIGURE 3.1

THEOREM 2.4. For any graph $G, \operatorname{sc}(G) \leq \beta(G)-\kappa(G)$.

Proof. For every $S \subset V(G)$, we have $c(G-S) \leq \beta(G)$. If $S$ is a cutset, then $|S| \geq$ $\kappa(G)$ and $c(G-S)-|S| \leq \beta(G)-\kappa(G)$. So

$$
\max _{S}\{c(G-S)-|S|\} \leq \beta(G)-\kappa(G) .
$$

The proof is completed.

3. Binomial trees and scattering number. In this section, we consider the binomial tree $B_{n}$ (Figure 3.1) (see [2]). The binomial tree $B_{n}$ is an ordered tree defined recursively. The binomial tree $B_{0}$ consists of a single vertex. The binomial tree $B_{n}$ consists of two binomial trees $B_{n-1}$ that are linked together: the root of one is the leftmost child of the root of the other.

Now we give the scattering number of a binomial tree.

THEOREM 3.1. Let $n \geq 3$ be a positive integer. Then $\operatorname{sc}\left(B_{n}\right)=2^{n-2}$.

Proof. In Figure 3.1, we call the vertex $u$ top vertex of $B_{n}$. Let $r$ be the number of removing vertices of $B_{n}$. If we remove top vertex $u$ of $B_{n}$, then $B_{n-1}, B_{n-2}, \ldots, B_{1}, B_{0}$ are components. Hence the number of components is $n$. Now if we remove top vertex of $B_{n-1}$, then we obtain the components $B_{n-2}, B_{n-3}, \ldots, B_{1}, B_{0}$. Then we have $2(n-1)$ components. If we continue to remove the top vertex of each component, then we have two cases.

CASE 1. If $r=2^{i}$ where $0 \leq i \leq n-1$, then the number of remaining components is exactly $(n-i) 2^{i}$ where $0 \leq i \leq n-1$. Hence

$$
\operatorname{sc}\left(B_{n}\right)=\max _{0 \leq i \leq n-1}\left\{(n-i) 2^{i}-2^{i}\right\} .
$$

CASE 2. If $2^{i-1}<r<2^{i}$ where $2 \leq i \leq n-1$, then the number of remaining components is exactly $(n-(i-1)) 2^{i-1}+\left(r-2^{i-1}\right)(n-(i+1))$. Hence

$$
\operatorname{sc}\left(B_{n}\right)=\max _{2 \leq i \leq n-1}\left\{(n-(i-1)) 2^{i-1}+\left(r-2^{i-1}\right)(n-(i+1))-r\right\} .
$$

Now we can show that

$$
\max _{2 \leq i \leq n-1}\left\{(n-(i-1)) 2^{i-1}+\left(r-2^{i-1}\right)(n-(i+1))-r\right\} \leq \max _{0 \leq i \leq n-1}\left\{(n-i) 2^{i}-2^{i}\right\} .
$$


Then

$$
\operatorname{sc}\left(B_{n}\right)=\max _{0 \leq i<n}\left\{(n-i) 2^{i}-2^{i}\right\} .
$$

The function $(n-i) 2^{i}-2^{i}$ takes its maximum value at $i=\lceil n-1 / \ln 2-1\rceil$. It is obvious that $\lceil n-1 / \ln 2-1\rceil=n-2$ for every $n \geq 3$. Hence if we substitute this value in the function $(n-i) 2^{i}-2^{i}$, then the proof is completed.

DEFINITION 3.2. The tensor product of two graphs $G=(V(G), E(G))$ and $H=$ $(V(H), E(H))$, denoted by $G \otimes H$, has the vertex set $V(G) \times V(H)$, the Cartesian product of $V(G)$ and $V(H)$, and an edge between vertices $(x, y)$ and $(u, v)$, if and only if $\{x, u\} \in E(G)$ and $\{y, v\} \in E(H)$.

THEOREM 3.3. Let $m \geq 4$ and $n \geq 4$ be positive integers. Then

$$
\operatorname{sc}\left(B_{m} \otimes B_{n}\right)=\max \left\{2^{n-1}, 2^{m-1}\right\} .
$$

Proof. The graph $B_{m} \otimes B_{n}$ has the graphs $B_{m}$ and $B_{n}$ as subgraphs. We consider these graphs, respectively. Let $r$ be the number of removing vertices from $B_{m} \otimes B_{n}$. Then we have two cases, depending on $B_{m}$ or $B_{n}$.

CASE 1. Let $u_{1}, u_{2}, \ldots, u_{2^{m}}$ be the vertices of $B_{m}$ and let $v$ be the top vertex of $B_{n}$. If we remove the vertices $u_{i} v\left(i=1,2, \ldots, 2^{m}\right)$, then the remaining components are $B_{m} \otimes B_{n-1}, B_{m} \otimes B_{n-2}, \ldots, B_{m} \otimes B_{1}$, and $2^{m} B_{0}$. Now let the top vertex of $B_{n-1}$ be $v^{\prime}$. If we remove the vertices $u_{i} v^{\prime}\left(i=1,2, \ldots, 2^{m}\right)$, then we obtain the components $B_{m} \otimes$ $B_{n-2}, \ldots, B_{m} \otimes B_{1}$ and $2^{m} B_{0}$. If we continue to remove the vertices as mentioned above, then we obtain the following cases for $r$.

(a) If $r=2^{m} 2^{i}$ where $0 \leq i \leq n-2$, then the number of components is $(n-i) 2^{i}+2^{m} 2^{i}$ and

$$
\operatorname{sc}\left(B_{m} \otimes B_{n}\right)=\max _{0 \leq i \leq n-2}\left\{2^{i}(n-i)\right\}
$$

(b) If $r=k 2^{m}$ where $2^{i-1}<k<2^{i}$ and $2 \leq i \leq n-2$, then the number of components is $(n-(i-1)) 2^{i-1}+\left(k-2^{i-1}\right)(n-(i+1))+2^{m} k$ and

$$
\operatorname{sc}\left(B_{m} \otimes B_{n}\right)=\max _{2^{i-1}<k<2^{i}, 2 \leq i \leq n-2}\left\{2^{i}+k(n-i-1)\right\} .
$$

(c) If $r=k 2^{m}$ and $2^{n-2}+1 \leq k \leq 2^{n-1}$, then the number of components is $k 2^{m}+$ $2\left(2^{n-1}-k\right)$ and

$$
\operatorname{sc}\left(B_{m} \otimes B_{n}\right)=\max _{2^{n-2}+1 \leq k \leq 2^{n-1}}\left\{2\left(2^{n-1}-k\right)\right\} .
$$

But we can show that

$$
\begin{aligned}
\max _{2^{i-1}<k<2^{i}, 2 \leq i \leq n-2}\left\{2^{i}+k(n-i-1)\right\} & \leq \max _{0 \leq i \leq n-2}\left\{2^{i}(n-i)\right\}, \\
\max _{2^{n-2}+1 \leq k \leq 2^{n-1}}\left\{2\left(2^{n-1}-k\right)\right\} & \leq \max _{0 \leq i \leq n-2}\left\{2^{i}(n-i)\right\} .
\end{aligned}
$$


Consequently, $\operatorname{sc}\left(B_{m} \otimes B_{n}\right)=\max _{0 \leq i \leq n-2}\left\{2^{i}(n-i)\right\}$. The function $2^{i}(n-i)$ takes its maximum value at $i=\lceil n-1 / \ln 2\rceil$. It is obvious that $\lceil n-1 / \ln 2\rceil=n-1$ for every $n \geq 4$ and so

$$
\operatorname{sc}\left(B_{m} \otimes B_{n}\right)=2^{n-1} .
$$

CASE 2. Let $v_{1}, v_{2}, \ldots, v_{2^{n}}$ be the vertices of $B_{n}$ and let $u$ be the top vertex of $B_{m}$. If we remove the vertices $u v_{i}\left(i=1,2, \ldots, 2^{n}\right)$, then the remaining components are $B_{m-1} \otimes B_{n}, B_{m-2} \otimes B_{n}, \ldots, B_{1} \otimes B_{n}$, and $2^{n} B_{0}$. Now let the top vertex of $B_{m-1}$ be $u^{\prime}$. If we remove the vertices $u^{\prime} v_{i}\left(i=1,2, \ldots, 2^{n}\right)$, then we obtain the components $B_{m-2} \otimes$ $B_{n}, \ldots, B_{1} \otimes B_{n}$ and $2^{n} B_{0}$. If we continue to remove the vertices as mentioned above, then we obtain the following cases for $r$.

(a) If $r=2^{n} 2^{i}$ where $0 \leq i \leq m-2$, then the number of components is $(m-i) 2^{i}+$ $2^{n} 2^{i}$ and

$$
\operatorname{sc}\left(B_{m} \otimes B_{n}\right)=\max _{0 \leq i \leq m-2}\left\{2^{i}(m-i)\right\} .
$$

(b) If $r=k 2^{n}$ where $2^{i-1}<k<2^{i}$ and $2 \leq i \leq m-2$, then the number of components is $(m-(i-1)) 2^{i-1}+\left(k-2^{i-1}\right)(m-(i+1))+2^{n} k$ and

$$
\mathrm{sc}\left(B_{m} \otimes B_{n}\right)=\max _{2^{i-1}<k<2^{i}, 2 \leq i \leq m-2}\left\{2^{i}+k(m-i-1)\right\} .
$$

(c) If $r=k 2^{n}$ and $2^{m-2}+1 \leq k \leq 2^{m-1}$, then the number of components is $k 2^{n}+$ $2\left(2^{m-1}-k\right)$ and

$$
\operatorname{sc}\left(B_{m} \otimes B_{n}\right)=\max _{2^{m-2}+1 \leq k \leq 2^{m-1}}\left\{2\left(2^{m-1}-k\right)\right\} .
$$

But we can show that

$$
\begin{aligned}
\max _{2^{i-1}<k<2^{i}, 2 \leq i \leq m-2}\left\{2^{i}+k(m-i-1)\right\} & \leq \max _{0 \leq i \leq m-2}\left\{2^{i}(m-i)\right\}, \\
\max _{2^{m-2}+1 \leq k \leq 2^{m-1}}\left\{2\left(2^{m-1}-k\right)\right\} & \leq \max _{0 \leq i \leq m-2}\left\{2^{i}(m-i)\right\} .
\end{aligned}
$$

Consequently, $\operatorname{sc}\left(B_{m} \otimes B_{n}\right)=\max _{0 \leq i \leq m-2}\left\{2^{i}(m-i)\right\}$. The function $2^{i}(m-i)$ takes its maximum value at $i=\lceil m-1 / \ln 2\rceil$. It is obvious that $\lceil m-1 / \ln 2\rceil=m-1$ for every $m \geq 4$ and so

$$
\operatorname{sc}\left(B_{m} \otimes B_{n}\right)=2^{m-1} .
$$

By (3.10) and (3.15) we have $\operatorname{sc}\left(B_{m} \otimes B_{n}\right)=\max \left\{2^{m-1}, 2^{n-1}\right\}$.

The proof is completed.

Definition 3.4. Let $G_{1}$ and $G_{2}$ be two graphs. The union $G=G_{1} \cup G_{2}$ has $V(G)=$ $V\left(G_{1}\right) \cup V\left(G_{2}\right)$ and $E(G)=E\left(G_{1}\right) \cup E\left(G_{2}\right)$. The join is denoted $V\left(G_{1}\right)+V\left(G_{2}\right)$ and consists of $V\left(G_{1}\right) \cup V\left(G_{2}\right)$ and all edges joining $V\left(G_{1}\right)$ with $V\left(G_{2}\right)$. For three or more disjoint graphs $G_{1}, G_{2}, \ldots, G_{n}$, the sequential join $G_{1}+G_{2}+\cdots+G_{n}$ is $\left(G_{1}+G_{2}\right) \cup\left(G_{2}+\right.$ $\left.G_{3}\right) \cup \cdots \cup\left(G_{n-1}+G_{n}\right)$. 
THEOREM 3.5. If $n$ is an even number, then $\operatorname{sc}\left(B_{0}+B_{1}+\cdots+B_{n}\right)=4 / 3-2^{n} / 3$.

Proof. To prove this theorem we have two cases.

CASE 1. If we remove the vertices of graphs $B_{1}, B_{3}, \ldots, B_{n-1}$, then the remaining components are $B_{0}, B_{2}, \ldots, B_{n}$ and the number of removing vertices is $\sum_{i=1}^{n / 2}\left|V\left(B_{2 i-1}\right)\right|=$ $\sum_{i=1}^{n / 2} 2^{2 i-1}$. Moreover, we must delete $2^{2(i-1)}$ more vertices from each $B_{2 i}$ where $0<$ $i \leq n / 2$ (except $B_{0}$ ). Hence $2 * 2^{2(i-1)}$ components are obtained from each $B_{2 i}$ where $0<i \leq n / 2$ (except $B_{0}$ ). Then the number of removing vertices is exactly

$$
|S|=\sum_{i=1}^{n / 2} 2^{2 i-1}+\sum_{i=1}^{n / 2} 2^{2(i-1)}
$$

and the number of components is exactly

$$
c\left(\left(B_{0}+B_{1}+\cdots+B_{n}\right)-S\right)=2 \sum_{i=1}^{n / 2} 2^{2(i-1)}+1 .
$$

So

$$
\operatorname{sc}\left(B_{0}+B_{1}+\cdots+B_{n}\right)=1-\frac{1}{4} \sum_{i=1}^{n / 2} 2^{2 i} .
$$

CASE 2. If we remove the vertices of graphs $B_{0}, B_{2}, \ldots, B_{n}$, then the remaining components are $B_{1}, B_{3}, \ldots, B_{n-1}$ and the number of removing vertices is $\sum_{i=0}^{n / 2}\left|V\left(B_{2 i}\right)\right|=$ $\sum_{i=0}^{n / 2} 2^{2 i}$. Moreover, we must delete $2^{2 i-3}$ more vertices from each $B_{2 i-1}$ where $1<$ $i \leq n / 2$ (except $B_{1}$ ). Hence $2 * 2^{2 i-3}$ components are obtained from each $B_{2 i-1}$ where $1<i \leq n / 2$ (except $B_{1}$ ). Then the number of removing vertices is exactly

$$
|S|=\sum_{i=1}^{n / 2} 2^{2(i-1)}+\sum_{i=0}^{n / 2} 2^{2 i}
$$

and the number of components is exactly

$$
c\left(\left(B_{0}+B_{1}+\cdots+B_{n}\right)-S\right)=2 \sum_{i=2}^{n / 2} 2^{2 i-3}+1 .
$$

So

$$
\operatorname{sc}\left(B_{0}+B_{1}+\cdots+B_{n}\right)=-5-\sum_{i=2}^{n / 2} 2^{2 i} .
$$

By (3.18) and (3.21), we have

$$
\operatorname{sc}\left(B_{0}+B_{1}+\cdots+B_{n}\right)=\max _{i}\left\{1-\frac{1}{4} \sum_{i=1}^{n / 2} 2^{2 i},-5-\sum_{i=2}^{n / 2} 2^{2 i}\right\} .
$$

Since $\sum a^{t}=a^{t} /(a-1)+c(t)$ where $a \neq 1$, we have

$$
\operatorname{sc}\left(B_{0}+B_{1}+\cdots+B_{n}\right)=\max _{i}\left\{\frac{4}{3}-\frac{2^{n}}{3}, \frac{1}{3}-\frac{4}{3} 2^{n}\right\}=\frac{4}{3}-\frac{2^{n}}{3} \text {. }
$$

The proof is completed. 
THEOREM 3.6. If $n$ is an odd number, then $\operatorname{sc}\left(B_{0}+B_{1}+\cdots+B_{n}\right)=2 / 3-2^{n} / 3$.

Proof. The proof follows directly from Theorem 3.5.

\section{REFERENCES}

[1] V. Chvátal, Tough graphs and Hamiltonian circuits, Discrete Math. 5 (1973), 215-228.

[2] T. H. Cormen, C. E. Leiserson, and R. L. Rivest, Introduction to Algorithms, 4th ed., MIT Press, Massachusetts, 1990.

[3] J. S. Deogun, D. Kratsch, and G. Steiner, 1-Tough cocomparability graphs are Hamiltonian, Discrete Math. 170 (1997), 99-106.

[4] H. A. Jung, On a class of posets and the corresponding comparability graphs, J. Combinatorial Theory Ser. B 24 (1978), 125-133.

[5] D. Kratsch, T. Kloks, and H. Müller, Measuring the vulnerability for classes of intersection graphs, Discrete Appl. Math. 77 (1997), 259-270.

[6] J. Lehel, The path partition of cocomparability graphs, unpublished, 1991.

AlPAy KiRLANGiÇ: DePARTMENT OF MATHEMATICS, EgE UNIVERSITY, 35100 BORNOVA-IZMIR, TURKEY

E-mail address: a7pkir@sci.ege.edu.tr 


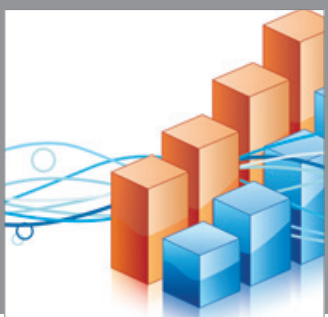

Advances in

Operations Research

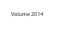

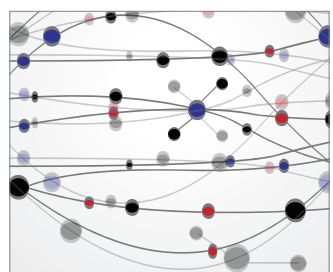

\section{The Scientific} World Journal
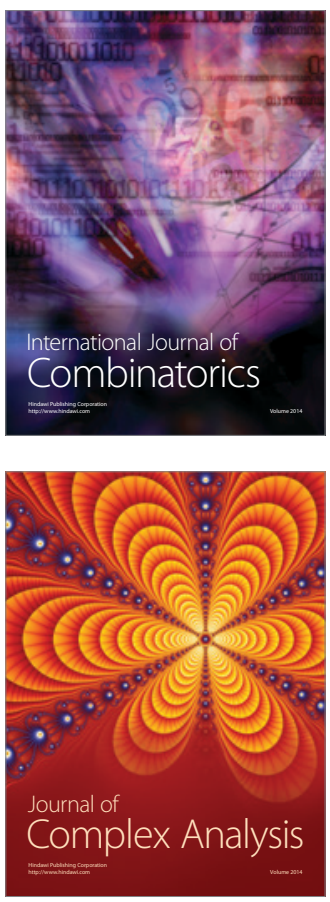

International Journal of

Mathematics and

Mathematical

Sciences
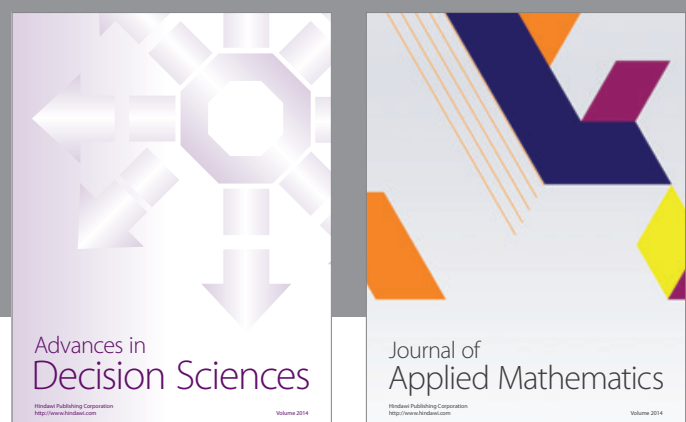

Journal of

Applied Mathematics
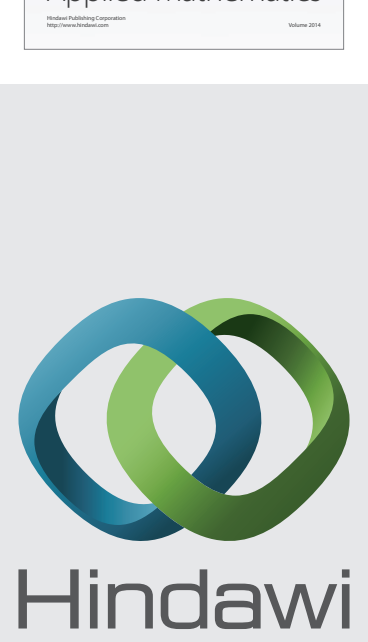

Submit your manuscripts at http://www.hindawi.com
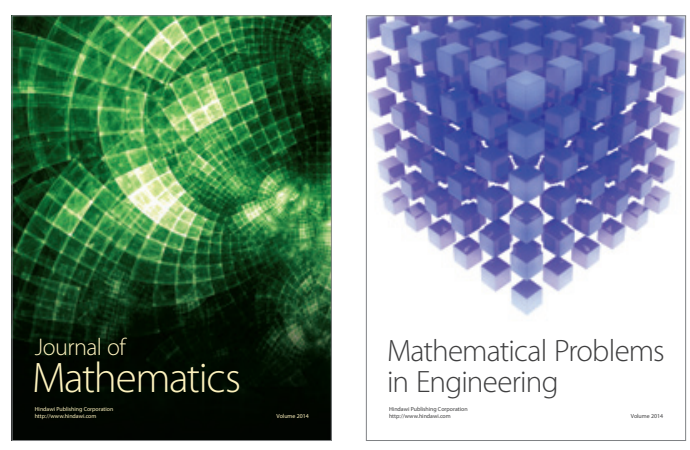

Mathematical Problems in Engineering
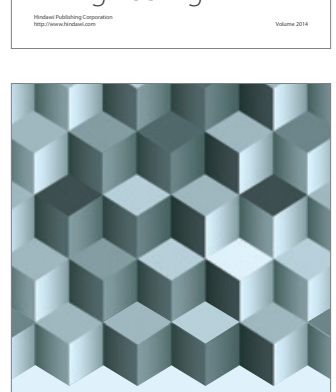

Journal of

Function Spaces
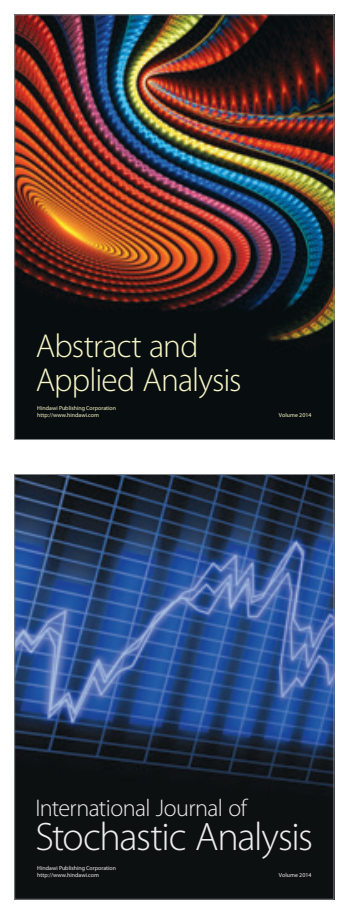

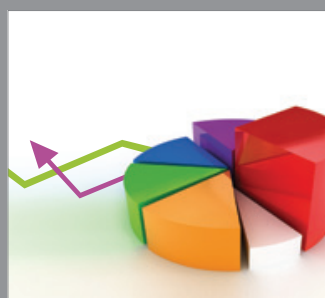

ournal of

Probability and Statistics

Promensencen
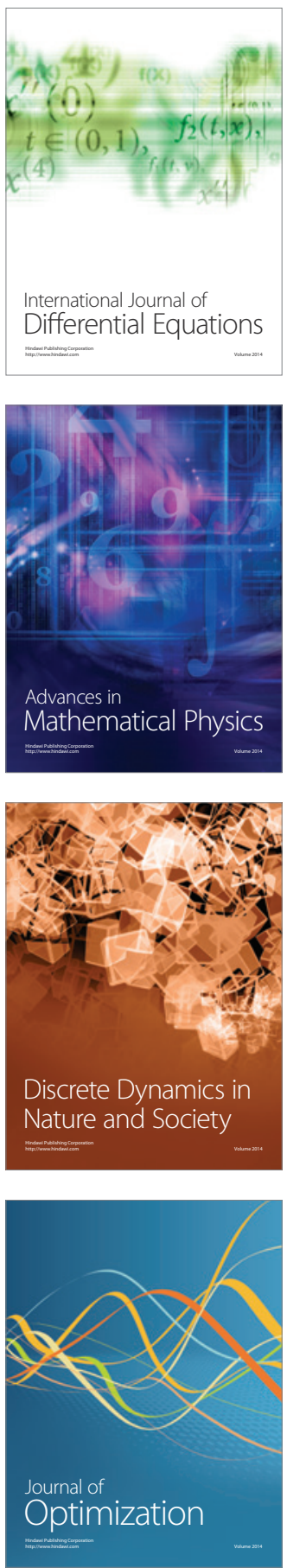